\title{
Smoothing techniques for decision-directed MIMO OFDM channel estimation
}

\section{P. Beinschob and U. Zölzer}

Department of Signal Processing and Communications, Helmut-Schmidt-Universität/University of the Federal Armed Forces, Hamburg, Holstenhofweg 85, 22043 Hamburg, Germany

\begin{abstract}
With the purpose of supplying the demand of faster and more reliable communication, multiple-input multiple-output (MIMO) systems in conjunction with Orthogonal Frequency Division Multiplexing (OFDM) are subject of extensive research. Successful Decoding requires an accurate channel estimate at the receiver, which is gained either by evaluation of reference symbols which requires designated resources in the transmit signal or decision-directed approaches. The latter offers a convenient way to maximize bandwidth efficiency, but it suffers from error propagation due to the dependency between the decoding of the current data symbol and the calculation of the next channel estimate. In our contribution we consider linear smoothing techniques to mitigate error propagation by the introduction of backward dependencies in the decision-based channel estimation. Designed as a post-processing step, frame repeat requests can be lowered by applying this technique if the data is insensitive to latency. The problem of high memory requirements of FIR smoothing in the context of MIMO-OFDM is addressed with an recursive approach that acquires minimal resources with virtual no performance loss. Channel estimate normalized mean square error and bit error rate (BER) performance evaluations are presented. For reference, a median filtering technique is presented that operates on the MIMO time-frequency grids of channel coefficients to reduce the peak-like outliers produced by wrong decisions due to unsuccessful decoding. Performance in terms of Bit Error Rate is compared to the proposed smoothing techniques.
\end{abstract}

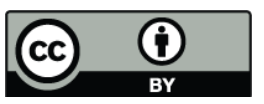

Correspondence to: $\mathrm{P}$. Beinschob (patric.beinschob@hsu-hamburg.de)

\section{Introduction}

MIMO OFDM systems are strong candidates for upcoming mobile networks. In Spatial Multiplexing transmission modes the channel capacity increases linearly with the number of transmit and receive antennas primary at the expense of higher SNR demands and algorithmic complexity in the receiver and detection algorithms (Foschini and Gans, 1998).

However, there can be observed large gaps between analytical derived capacity statements and realized data rates with proposed receiver designs. This is particularly true for mobile scenarios. Channel estimate errors tend to decrease the achievable rates because of the limited performance of the detection algorithms operating with channel estimates (Dall'Anese et al., 2009).

To acquire channel estimates with low errors a large number of reference symbols or pilots would be necessary. Exclusive bandwidth has to be dedicated to reference symbols and is not available for data transmission, therefore the effective data rate is reduced by the amount of pilots which is not desirable. In mobile receivers the channel estimate is quickly outdated depending on the relative radial velocity to the sender (Marzetta and Hochwald, 1999). After a time duration, commonly known as the coherence time $T_{\mathrm{C}}$, the channel state must be assumed to be completely changed.

Cellular mobile networks in urban environment additionally experience multipath signal propagation often referred to street canyon scenarios and alike. So, Doppler shifts due to mobility vary on different propagation paths. Fading due to shadowing might occur suddenly and the channel estimation has to keep track.

The mentioned aspects and problems of mobile multiantenna broadband communication and the consequences for the channel estimation is addressed in this paper. To mitigate influences we propose a post-processing - smoothing - algorithm to refine the channel estimates acquired by a decisiondirected channel estimation (DDCE) and tracking algorithm.

Published by Copernicus Publications on behalf of the URSI Landesausschuss in der Bundesrepublik Deutschland e.V. 


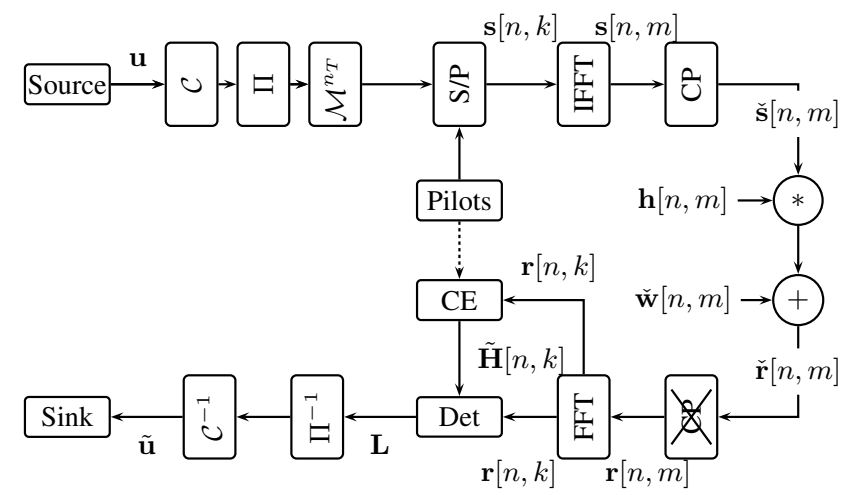

Fig. 1. MIMO-OFDM system model.

The DDCE based on Least Squares works well with a small number of pilot symbols in quasi-static scenarios.

The paper is organized as follows. The underlying system model in time and frequency-domain is presented in Sect. 2, followed by a brief review of the DDCE algorithm in Sect. 3. An approach to introduce linear smoothing techniques in the context of MIMO-OFDM channel estimation is taken in Sect. 4.1 and a memory-reduced version shown in Sect. 4.2. An alternative, non-linear filtering method is discussed in Sect. 4.3. Illustrating simulation results are given in Sect. 5 and finally a conclusion is drawn.

\section{System model and structure}

The vector of received values $\boldsymbol{r}$ at the time sample $m$ of a MIMO system is the superposition of $L \cdot n_{\mathrm{T}}$ previously sent samples and the current $n_{\mathrm{T}}$ samples, where $L+1$ is the length of the sampled channel impulse response and $n_{\mathrm{T}}$ is the number of transmit antennas. It is given by

$\boldsymbol{r}[m]=\sum_{l=0}^{L} \mathbf{h}[l, m] \cdot \boldsymbol{s}[m-l]+\tilde{\boldsymbol{w}}[m]$,

where $s[m]$ denotes the current vector of symbols of each transmit antenna, $\boldsymbol{w}$ is an identically, independently distributed (iid) additive white Gaussian noise term and $\mathbf{h}[l, m]$ is the MIMO channel matrix in delay and time domain, indexed with $l$ respectively $m$. The past sent samples are denoted by $s[m-l]$, for $l \neq 0, l \leq L$. The data symbols of the $K$ subcarriers are modulated by an inverse Fast Fourier Transform (IFFT). In simulations every value corresponding to a transmit antenna of the resulting vectors is transmitted using the formula above. The data symbols itself are drawn from an $M$-order QAM modulation alphabet $\mathbb{S}$. The mapping, denoted by $\mathcal{M}\{\cdot\}$, modulates $\kappa=\log _{2} M$ bits to a QAM symbol. This is done consecutively for all $n_{\mathrm{T}}$ send streams/layers. The QAM constellations are considered power-normalized to simplify notation.
In frequency domain the system model in Eq. (1) can be described as

$\boldsymbol{r}[n, k]=\mathbf{H}[n, k] \cdot \boldsymbol{s}[n, k]+\boldsymbol{w}[n, k]$,

where $n$ denotes the time index of an OFDM symbol and $k$ its subcarrier index. The vectors $\boldsymbol{r}[n, k]$ and $\boldsymbol{w}[n, k]$ are of dimension $n_{\mathrm{R}} \times 1, \boldsymbol{s}[n, k]$ of $n_{\mathrm{T}} \times 1$ and the matrix $\mathbf{H}[n, k]$ of $n_{\mathrm{R}} \times n_{\mathrm{T}}$, at which $n_{\mathrm{R}}$ is the number of receive antennas. In simulations the time domain MIMO channel coefficients $h_{r, t}[l, m], r=1, \ldots, n_{\mathrm{R}}, t=1, \ldots, n_{\mathrm{T}}$ are modeled using the 3GPP spatial model which was developed to evaluate receiver algorithms in MIMO scenarios (3rd Generation Partnership Project, 3GPP). The superposed received signals are transferred back into the frequency domain with the help of a FFT, resulting in the vectors $\boldsymbol{r}[n, k]$ of Eq. (2). Perfect synchronization is assumed and total avoidance of block interference, i.e. the cyclic prefix is longer than the maximum delay path. The system's performance is evaluated in terms of bit error rates determined from hard decided channel decoder output. The channel decoder operates on soft information in form of channel log-likelihood ratios (LLRs), L. A block diagram depicting the system model is given in Fig. 1.

\section{Decision-directed Recursive Least Squares (RLS) channel estimation}

MIMO-RLS algorithm estimates auto- and cross-correlation matrices $\boldsymbol{\Phi}$ resp. $\boldsymbol{\theta}$, time adaptive with forgetting factor $\xi$ as described by Kay (1993). The channel estimation is done on each subcarrier $k$ individually.

$\begin{aligned} \boldsymbol{\Phi}[n, k] & =\xi \cdot \boldsymbol{\Phi}[n-1, k]+\boldsymbol{s}[n, k] \cdot \boldsymbol{s}^{\mathrm{H}}[n, k] \\ \boldsymbol{\theta}[n, k] & =\boldsymbol{\xi} \cdot \boldsymbol{\theta}[n-1, k]+\boldsymbol{s}[n, k] \cdot \boldsymbol{r}^{\mathrm{H}}[n, k]\end{aligned}$

An estimate of the channel matrix is obtained as follows:

$\tilde{\mathbf{H}}[n, k]=\left(\boldsymbol{\Phi}^{-1}[n, k] \cdot \boldsymbol{\theta}[n, k]\right)^{\mathrm{H}}$.

From the MIMO bit-wise log-likelihood ratio detection output, that is soft information of channel output $\mathbf{L}$, reconstructed send vectors are available. After the pilot sequence of length $N_{P}$ in the preamble of the frame is processed, the reconstructed send vectors

$\tilde{\boldsymbol{s}}[n]=\mathcal{M}^{n_{\mathrm{T}}}\{\operatorname{sgn}\{\mathbf{L}\}\}, \quad \forall n>N_{\mathrm{P}}$.

are used to further refine the estimate of the auto- and crosscorrelation matrices thus tracking the time-variable channel, as indicated by the switch in Fig. 2 (Akhtman and Hanzo, 2007). The coherence time of the channel determines the usability of the collected samples. Old samples describing an obsolete channel state should be omitted. This is accomplished by the introduction of the forgetting factor $\xi$. For the sake of notational simplicity the symbol $s$ is used for pilot vectors as well as the reconstructed send vectors omitting the tilde. 


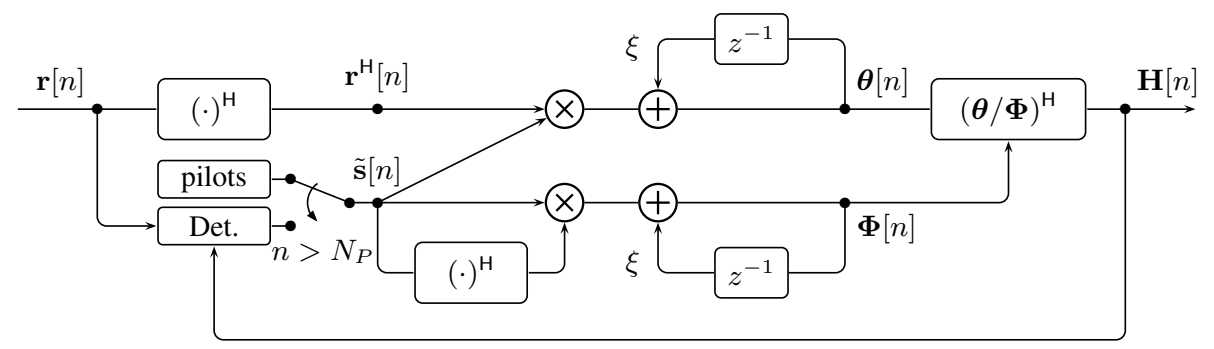

Fig. 2. Signal flow diagram for decision directed RLS channel estimation and tracking algorithm.

\section{Smoothing techniques}

\subsection{RLS un-rolling}

Post-processing after the RLS requires access to the spatial correlation samples for each OFDM symbol and subcarrier

$$
\begin{gathered}
\check{\boldsymbol{\Phi}}[n, k]=\boldsymbol{s}[n, k] \cdot \boldsymbol{s}^{\mathrm{H}}[n, k], \\
\check{\boldsymbol{\theta}}[n, k]=\boldsymbol{s}[n, k] \cdot \boldsymbol{r}^{\mathrm{H}}[n, k] .
\end{gathered}
$$

Considering a $n_{\mathrm{T}} \times n_{\mathrm{R}}$ MIMO OFDM system with $N_{\mathrm{S}}$ OFDM symbols per frame and $K$ subcarriers, the dimension of the arrays is $n_{\mathrm{T}} \times n_{\mathrm{R}} \times K \times N_{\mathrm{S}}$.

The weighted expectation value in Eqs. (3) and (4) is then

$$
\begin{aligned}
\boldsymbol{\Phi}[n, k] & =\xi \cdot \boldsymbol{\Phi}[n-1, k]+\check{\boldsymbol{\Phi}}[n, k], \\
\boldsymbol{\theta}[n, k] & =\xi \cdot \boldsymbol{\theta}[n-1, k]+\check{\boldsymbol{\theta}}[n, k] .
\end{aligned}
$$

It can be re-formulated as a matrix vector-multiplication. The weighting factors are exponentially decreasing with increasing sample index $n$ therefore they constitute a lower triangular matrix

$$
\boldsymbol{\Xi}=\left(\begin{array}{ccccc}
1 & 0 & \cdots & & \\
\xi & 1 & 0 & \cdots & \\
\xi^{2} & \xi & 1 & 0 & \cdots \\
\vdots & \vdots & \vdots & \ddots & 0 \\
\xi^{N_{S}-1} & \xi^{N_{S}-2} & \xi^{N_{S}-3} & \cdots & 1
\end{array}\right)
$$

The weighting along the time dimension with the forgetting factor matrix can be applied independently on the other dimensions

$\check{\boldsymbol{\Phi}}_{t, r, k}^{\prime}=\mathbf{\Xi} \cdot \check{\boldsymbol{\Phi}}_{t, r, k} \quad \forall t, r, k$

and analogue

$$
\check{\boldsymbol{\theta}}_{t, r, k}^{\prime}=\boldsymbol{\Xi} \cdot \check{\boldsymbol{\theta}}_{r, r, k} .
$$

With matrices (12) and (13) the ordinary channel estimate can be calculated as well

$$
\tilde{\mathbf{H}}[n, k]=\left(\check{\boldsymbol{\Phi}}^{\prime-1}[n, k] \cdot \check{\boldsymbol{\theta}}^{\prime}[n, k]\right)^{\mathrm{H}} .
$$

The smoothing algorithm can be derived by minimizing the modified cost function

$\check{J}[n, k]=\sum_{\tilde{n}=1}^{N_{S}} \xi^{\left|N_{\mathrm{S}}-\tilde{n}\right|} \cdot \mathbf{e}^{\mathrm{H}}[\tilde{n}, n, k] \cdot \mathbf{e}[\tilde{n}, n, k]$

with

$\mathbf{e}[\tilde{n}, n, k]=\boldsymbol{r}[\tilde{n}, k]-\tilde{\mathbf{H}}[n, k] \cdot s[\tilde{n}, k]$.

As a consequence it follows a transition for the weighting matrix to be fully occupied

$$
\mathbf{\Xi}^{\prime}=\left(\begin{array}{cccccc}
1 & \xi & \xi^{2} & \xi^{3} & \ldots & \xi^{N_{\mathrm{S}}-1} \\
\xi & 1 & \xi & \xi^{2} & \cdots & \xi^{N_{\mathrm{S}}-2} \\
\xi^{2} & \xi & 1 & \xi & \cdots & \xi^{N_{\mathrm{S}}-3} \\
\xi^{3} & \xi^{2} & \xi & 1 & \cdots & \xi^{N_{\mathrm{S}}-4} \\
\vdots & \vdots & \vdots & \vdots & \ddots & \vdots \\
\xi^{N_{\mathrm{S}}-1} & \xi^{N_{\mathrm{S}}-2} & \xi^{N_{\mathrm{S}}-3} & \xi^{N_{\mathrm{S}}-4} & \cdots & 1
\end{array}\right) .
$$

Smoothing weighting reflecting mobility induced channel time variance and effectively doubles the number of samples available for estimating the auto- and crosscorrelation matrices thus lowering the estimation error. Obviously, in this formulation arbitrary window functions can be applied as well instead of exponentially weighting factors in $\mathbf{\Xi}^{\prime}$. So smoothing weighting is applied by evaluating

$\check{\boldsymbol{\Phi}}_{t, r, k}^{\prime \prime}=\mathbf{\Xi}^{\prime} \cdot \check{\boldsymbol{\Phi}}_{t, r, k}$,

$\check{\boldsymbol{\theta}}_{t, r, k}^{\prime \prime}=\mathbf{\Xi}^{\prime} \cdot \check{\boldsymbol{\theta}}_{t, r, k}$,

The smoothed channel estimate for the unrolling $(\mathrm{U})$ is yielded by

$$
\tilde{\mathbf{H}}_{S}^{\mathrm{U}}[n, k]=\left(\check{\boldsymbol{\Phi}}^{\prime \prime-1}[n, k] \cdot \check{\boldsymbol{\theta}}^{\prime \prime}[n, k]\right)^{\mathrm{H}} .
$$

\subsection{Recursive smoothing}

The unrolling approach consumes a huge amount of memory impeding practical receiver implementations. Therefore a reduction of memory requirements is desirable. It is possible to reformulate the algorithm again into a recursive form (Kashima et al., 2006). Defining 


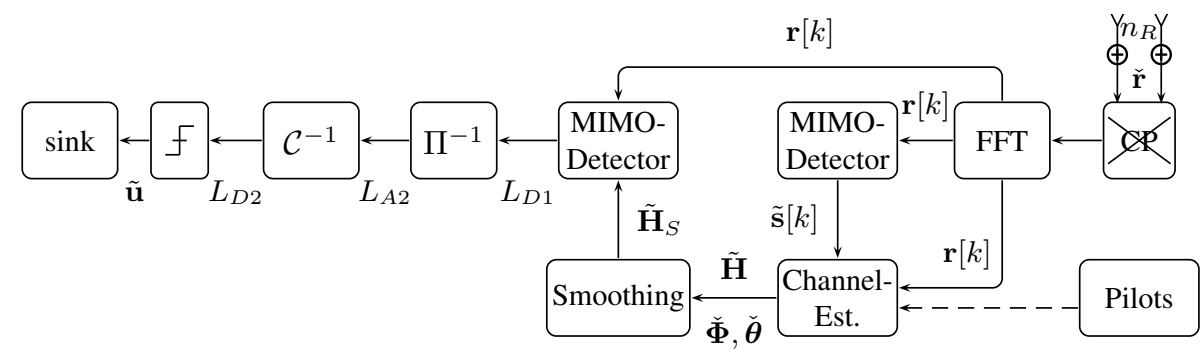

Fig. 3. Receiver structure with post processing of channel estimation with discussed smoothing techniques.

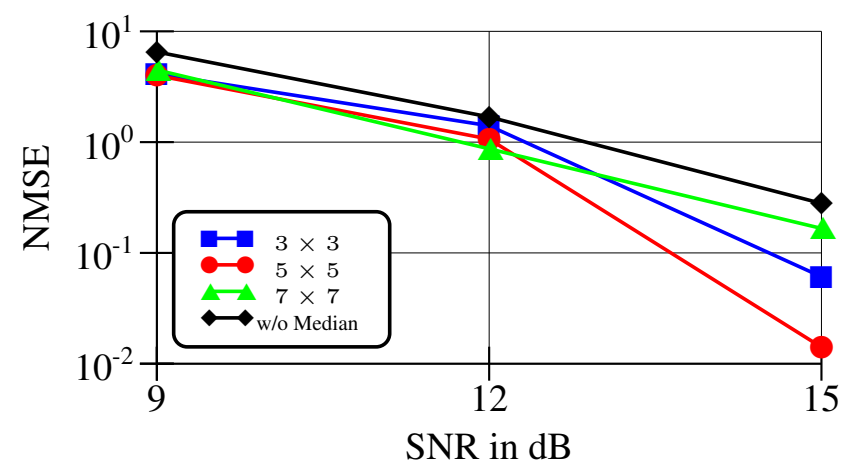

Fig. 4. NMSE comparison of different kernel sizes for Median Filtering in a time-variable channel with simulated mobile terminal velocity of $10 \mathrm{~m} \mathrm{~s}^{-1}$.

$\begin{aligned} \boldsymbol{\Phi}_{\mathrm{S}}[n, k] & =\sum_{\tilde{n}=1}^{N_{\mathrm{S}}} \xi^{\left|N_{\mathrm{S}}-\tilde{n}\right|} \cdot s[\tilde{n}, k] \cdot \boldsymbol{s}^{\mathrm{H}}[\tilde{n}, k], \\ \boldsymbol{\theta}_{\mathrm{S}}[n, k] & =\sum_{\tilde{n}=1}^{N_{\mathrm{S}}} \xi^{\left|N_{\mathrm{S}}-\tilde{n}\right|} \cdot \boldsymbol{s}[\tilde{n}, k] \cdot \boldsymbol{r}^{\mathrm{H}}[\tilde{n}, k],\end{aligned}$

it is possible to calculate the smoothed inverse autocorrelation matrix $\mathbf{P}_{\mathrm{S}}=\boldsymbol{\Phi}_{\mathrm{S}}^{-1}$ recursive using the already estimated inverse autocorrelation matrix $\boldsymbol{\Phi}$,

$\mathbf{P}_{\mathrm{S}}[n, k]=\mathbf{P}[n, k]+\xi^{2} \cdot\left[\mathbf{P}_{\mathrm{S}}[n+1, k]-\xi^{-1} \cdot \mathbf{P}[n, k]\right]$.

So a smoothed channel estimate can also be calculated using the previously estimated channel estimate $\tilde{\mathbf{H}}$ by evaluating

$\tilde{\mathbf{H}}_{\mathrm{S}}^{\mathrm{R}}[n, k]=\tilde{\mathbf{H}}[n, k]+\xi \cdot\left[\tilde{\mathbf{H}}_{\mathrm{S}}[n+1, k]-\tilde{\mathbf{H}}[n, k]\right]$,

where $n=N_{\mathrm{S}}, \ldots, 1$ and it is initialized by setting $\tilde{\mathbf{H}}_{\mathrm{S}}\left[N_{\mathrm{S}}, k\right]=\tilde{\mathbf{H}}\left[N_{\mathrm{S}}, k\right]$.

\subsection{Time-frequency median filtering}

Decision-directed channel estimation is prone to error propagation and wrong decisions may lead to impulse-like outliers in the channel estimate. As median filtering is especially suited for impulse noise it is investigated in this context as an alternative to the above discussed approaches to smoothing.

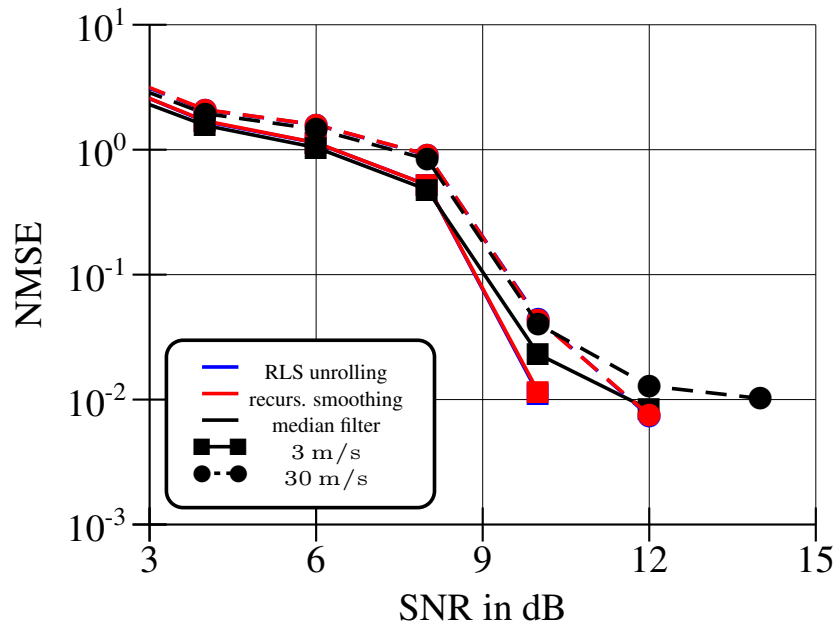

Fig. 5. NMSE comparison of different kernel sizes for Median Filtering in a time-variable channel with simulated mobile terminal velocity of $10 \mathrm{~m} \mathrm{~s}^{-1}$.

This non-linear filtering is applied on the $n_{\mathrm{T}} \cdot n_{\mathrm{R}}$ timefrequency grids resp. time-variant channel transfer functions. An important parameter is the filter kernel size. Often used sizes in image processing are $3 \times 3,5 \times 5$ or $7 \times 7$ samples. The optimal kernel size depends on the coherence time and coherence bandwidth respectively subcarriers.

\section{Simulation results}

The comparative simulations were conducted using a $4 \times 4$ MIMO-OFDM system configuration with $K=128$ subcarriers and a cyclic prefix length $L=6$ and two velocity setups $\left(3 \mathrm{~m} \mathrm{~s}^{-1}\right.$ and $\left.30 \mathrm{~m} \mathrm{~s}^{-1}\right)$. For a realistic channel the $3 \mathrm{GPP}$ Spatial Channel Model was used with time-variant impulse responses. Uniformly distributed bits were coded using an irregular LDPC code with design code rate of $1 / 2$, interleaved and 4-QAM modulated. LDPC codes are chosen because of high codeword distance and parallelisable decoder structure (Richardson et al., 2001). They are also employed in the IEEE 802.11n Standard for MIMO systems. 


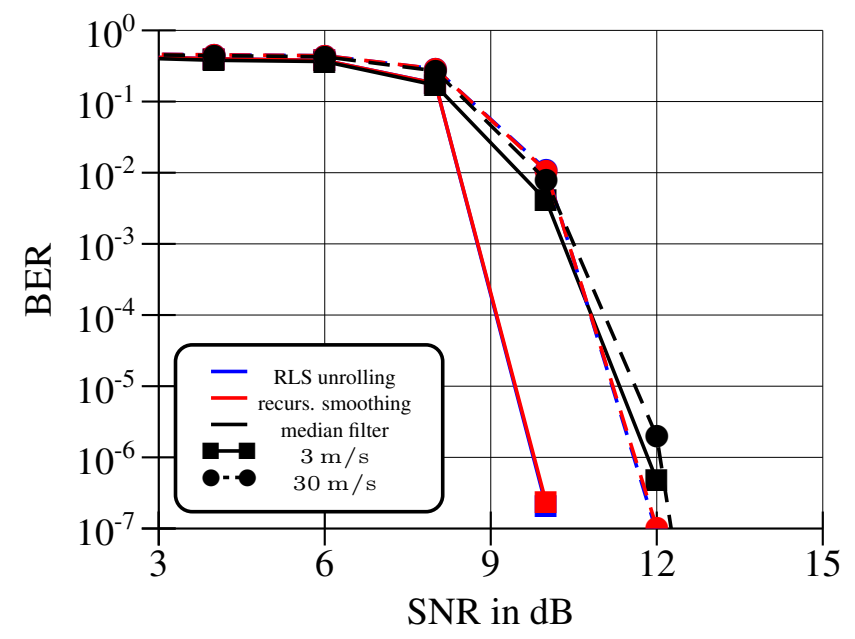

Fig. 6. Comparison of Bit Error Rates for discussed smoothing techniques.

For different median kernel sizes simulations had been conducted and the results in terms of NMSE are depicted in Fig. 4. A kernel size of $5 \times 5$ resulted in lowest average channel estimation errors which was then used in the overall comparative simulations.

Results in terms of channel estimation error measured in normalised mean squared error (NMSE) are presented in Fig. 5. A turbo-like cliff region (ten Brink, 2000) around $9 \mathrm{~dB}$ was observed. For SNR below this threshold the detector could not reproduce the send vectors reliable therefore error propagation in the decision-directed channel estimation algorithm started rendering complete frames useless and finally resulting in high channel estimation errors. Above the identified threshold the NMSE dramatically decreased. In this region performance differences for Median filtering are visible, whereas for the unrolling and the recursive Smoothing there were no significant differences in performance observable - the curves are almost identical, slightly better for the unrolling approach.

The Bit Error Rate (BER) versus SNR are depicted in Fig. 6. The results reflect the same tendency as the NMSE results did. In comparison to the linear smoothing techniques the performance of the Median filter is worse.

Reliable transmission is possible in the slowly time-variant case above $10 \mathrm{~dB}$ SNR employing one of the linear smoothing methods. It needs $12 \mathrm{~dB}$ or more with the Median filtering method. However, for the higher velocity the difference in minimum SNR is insignificant due to the high impact of intercarrier interference.

\section{Conclusions}

In this paper we have discussed smoothing techniques in the context of MIMO OFDM channel estimation. The output of a bandwidth-efficient decision-directed channel estimation and tracking algorithm is post-processed to improve the estimate's accuracy in mobile scenarios by effectively doubling the number of samples available for correlation matrix estimation. In a first approach, the modification of the RLS algorithm leads to a smoothing with high memory requirements. A memory efficient recursive smoothing algorithm has been presented having virtually no loss in NMSE or BER performance compared to the first approach in the conducted simulations with two velocity scenarios. As an alternative, median filtering is compared to the above mentioned. While it leads to comparable results for the high velocity case, a gap for the lower one reveals the disadvantage of the static kernel size in this method.

However, in future work these results has to be verified with measurement setups. Nevertheless the simulations indicate a potential to post-processing algorithms if the transmitted data is not sensitive to delays caused by the increased processing time and latency.

\section{References}

3rd Generation Partnership Project (3GPP): Spatial channel model for Multiple Input Multiple Output (MIMO) simulations, Tech. Spec. Group Radio Access Network, Tech. Report 25.996, Rel. 8.0.0, 2008.

Akhtman, J. and Hanzo, L.: Advanced Channel Estimation for MIMO-OFDM in Realistic Channel Conditions, IEEE International Conference on Communications, ICC '07, pp. 2528-2533, doi:10.1109/ICC.2007.418, 2007.

Dall'Anese, E., Assalini, A., and Pupolin, S.: On the Effect of Imperfect Channel Estimation upon the Capacity of Correlated MIMO Fading Channels, pp. 1-5, doi:10.1109/VETECS.2009. 5073728, 2009.

Foschini, G. J. and Gans, M. J.: On Limits of Wireless Communications in a Fading Environment when Using Multiple Antennas, Wireless Personal Communications: An International Journal, 6, 311-335, 1998.

Kashima, T., Fukawa, K., and Suzuki, H.: Recursive Least Squares Channel Estimator with Smoothing and Removing for IterativeMAP Receiver of MIMO-OFDM Mobile Communications, 7, 3052-3057, doi:10.1109/ICC.2006.255273, 2006.

Kay, S. M.: Estimation Theory, Fundamentals of statistical signal processing, vol. 1, Prentice Hall PTR, Upper Saddle River, NJ, 1993.

Marzetta, T. and Hochwald, B.: Capacity of a mobile multipleantenna communication link in Rayleigh flat fading, IEEE Transactions on Information Theory, 45, 139-157, doi:10.1109/18. 746779, 1999.

Richardson, T. J., Shokrollahi, M. A., and Urbanke, R. L.: Design of capacity-approaching irregular low-density parity-check codes, IEEE Trans. Inf. Theory, 47, 619-637, doi:http://dx.doi.org/10. 1109/18.910578, 2001.

ten Brink, S.: Designing Iterative Decoding Schemes with the Extrinsic Information Transfer Chart, AEU Int. J. Electron. Commun., pp. 389-398, 2000. 